BIOMEDICAL AND BIOSOCIAL ANTHROPOLOGY
$\begin{gathered}\text { Official Journal of the International Academy } \\ \text { of Integrative Anthropology } \\ \text { journal homepage: http://bba-journal.com }\end{gathered}$

\title{
Girth body sizes in men with psoriasis of different somatotypes
} Obadeh Bassam Abdel-Rahman Al-Qaraleh, Dmytrenko S. V., Kondratiuk A. I., Golubovsky I. A., Rekun T. O. National Pirogov Memorial Medical University, Vinnytsya, Ukraine

\section{ARTICLE INFO}

Received: 15 March 2021

Accepted: 16 April 2021

UDC: $616.517-055.1: 159.923$

\section{CORRESPONDING AUTHOR}

e-mail: dr.obadabassam@gmail.com Obadeh Bassam Abdel-Rahman Al-Qaraleh
Psoriasis, despite its seemingly simple clinic and course, is at the same time a complex socio-economic phenomenon that causes stigmatization of people with this disease. The use of constitutional markers to predict the risk and severity of psoriasis could be a promising way to alleviate this problem. The aim of the study was to establish and analyze the differences in girth body sizes between healthy and/or psoriatic Ukrainian men of different somatotypes depending on the severity of the disease. On the basis of the Military Medical Clinical Center of the Central Region and the Department of Skin and Venereal Diseases with a course of postgraduate education of National Pirogov Memorial Medical University, Vinnytsya, the determination of body sizes in men aged 22 to 35 years, suffering from psoriasis of mild ( $n=32)$ and severe $(n=68)$ course was carried out. Clinical assessment of the severity and area of psoriatic lesions was performed using the PASI index. Determination of somatotype was performed according to the Heath Carter method. As a control from the data bank of the research center of National Pirogov Memorial Medical University, Vinnytsya anthropometric data of 82 practically healthy men of the same age group were selected. Statistical processing of the obtained results was performed in the license package "Statistica 5.5" using non-parametric evaluation methods. Compared with healthy men of the corresponding somatotypes, it was found: in patients with mild and severe psoriasis men of mesomorphic somatotype - significantly higher, or a tendency to greater values of almost all girth body sizes (except foot girth, and only in patients with mild course - girth of forearms in the lower part); only in patients with mild psoriasis men of endomesomorphic somatotype - significantly higher, or a tendency to higher values of almost all girth body sizes (except for the girth of the foot and lower leg in the lower part). In patients with psoriasis men of endo-mesomorphic somatotype with a mild course of the disease significantly higher, or a tendency to greater values than in patients with a similar course men of mesomorphic somatotype shoulder girth in stressed and unstressed state, forearms, upper and lower arms thighs, hips, shins in the upper part, neck, waist and all the girths of the chest; and in patients with psoriasis men of mesomorphic somatotype with a severe course of the disease - only tendencies to greater values than in patients with a similar course men of endo-mesomorphic somatotype girth of thighs and shin in the upper part. In patients with mild psoriasis men, the differences in body sizes between mesomorphic and endo-mesomorphic somatotypes are similar to healthy men, but are more pronounced. When comparing the girth of the body between psoriasis patients Ukrainian men of the corresponding somatotypes, representatives of the mesomorphic somatotype with severe disease have significantly higher, or a tendency to greater values than with mild course, the girth values of the forearms at the top, neck, waist and all around chest, as well as the tendency to lower values of the girth of the shin. The established pronounced differences in the girth sizes of the body provide an opportunity to identify risk groups for the occurrence and features of psoriasis.

Keywords: psoriasis, girth body sizes, somatotype, men.

\section{Introduction}

The multifactorial nature of psoriasis and its prevalence are the reasons that encourage researchers to return again and again to study the nature of this brew, ways to prevent its occurrence and find optimal treatments. Scientists are still debating the key triggers responsible for the development of psoriasis.

It is common theory to combine external factors, triggers, which may include stress, bad habits, poor environmental 
conditions, insolation, etc. [8] and internal factors - genetic, responsible for Th17 cell activation, which include genes IL12B, IL23A, IL23R, TYK2, REL, TNIP1, NFKBIZ, TNFAIP3 and others [5]. However, a direct and reliable association of any gene with the development of psoriasis has not yet been found - data from studies on monozygotic twins vary from 35 to $72 \%$ of the association and has the highest indicators so far PSORS1 gene [8].

If we take into account the prevalence of psoriasis - it is strikingly different in different countries. In particular, there is a marked difference in prevalence according to the continent. Thus, the lowest prevalence rates are in the United Republic of Tanzania - $0.09 \%$ and Tunisia - $0.57 \%$, both countries are on the African continent. China has intermediate indicators where the prevalence according to various studies ranges from $0.12 \%$ to $1.49 \%$. Psoriasis is more common in Europe, with $0.73 \%$ in the United Kingdom and $2.90 \%$ in Italy. However, the highest rate is recorded in the United States and according to various publications ranges from $1.43 \%$ to $5.1 \%$ [15].

It should be noted that the above data apply to all age groups. If we study the prevalence of psoriasis according to different age groups, these priorities change significantly. In Italy, the prevalence among children is $2.1 \%$, and among adults only the prevalence in the United States is $0.91 \%$. The leader in terms of prevalence, if we take into account only the adult population, is Norway, with a rate of $8.5 \%$. At the same time, the prevalence of psoriasis among children in Taiwan is $0 \%$ [18], while in the general population is $0.235 \%$ [22].

In addition, the racial and ethnic component must be taken into account if the population of the country is diverse and includes different nationalities. If we take into account the example of the United States with an average prevalence of psoriasis of $3.2 \%$ (95\% confidence interval $\mathrm{Cl} 2.6 \%$ $3.7 \%$ ), ie 7.4 million sick people, the prevalence of the disease based on race has some differences: the prevalence among Caucasians is $3.6 \%(95 \% \mathrm{Cl} 2.7 \%$ $4.4 \%)$, among Africans $1.9 \%(95 \% \mathrm{Cl} 1.0 \%-2.8 \%)$, among Latinos $1.6 \%$ (95\% Cl $0.5 \%-2.8 \%$ ) [19].

Another important factor that motivates the study of psoriasis is its association with certain diseases. According to surveys of patients in Taiwan, psoriasis is associated with an increased incidence of hypertension (RR 1.51), diabetes (RR 1.64), rheumatoid arthritis ( $R R$ 3.02), systemic lupus erythematosus (RR 6, 16), asthma (RR 1.29), allergic rhinitis (RR 1.25) and other pathologies [22].

Psychological problems experienced by patients with psoriasis are another problem that affects the quality of life and work of these individuals. Psoriasis interferes with the full enjoyment of life by $82 \%$ of patients, and $88 \%$ of patients report one or another effect of their disease on the emotional state. $83 \%$ try to hide the manifestations of the disease, and $92 \%$ of the unemployed with psoriasis indicated the disease as the cause of unemployment. $49 \%$ of patients regularly missed work days due to certain manifestations of psoriasis [1].

According to Heller M. M. and co-authors, 37 to $78 \%$ of patients with psoriasis consider stress to be a trigger for exacerbation of their disease [11]. The vast majority of patients experience stigma due to their disease [12].

Patients with psoriasis in the United States incur additional costs of $\$ 2,284$ each year due to their disease, compared to $\$ 35.2$ billion annually in the country as a whole [23].

Thus, it becomes clear that a disease such as psoriasis is a global and multifaceted problem that requires a multidisciplinary approach to its solution. Anthropology is one of such disciplines that can be used to predict the occurrence and course of psoriasis, taking into account certain anthropometric indicators.

The aim of the study was to establish and analyze the differences in girth body sizes between healthy and/or psoriatic Ukrainian men of different somatotypes depending on the severity of the disease.

\section{Materials and methods}

According to the scheme of Bunak V. V. [4], on the basis of the Military Medical Clinical Center of the Central Region and the Department of Skin and Venereal Diseases with a course of postgraduate education National Pirogov Memorial Medical University, Vinnytsya, conducted anthropometric survey of men aged 22 to 35 years, who suffer from mild $(n=32)$ and severe $(n=68)$ psoriasis. Clinical assessment of the severity and area of psoriatic lesions was performed using the PASI index (Psoriasis Area and Severity Index) [2, 9]. Determination of somatotype was performed according to the method of Heath Carter [6].

As a control from the database of the research center of the National Pirogov Memorial Medical University, Vinnytsya selected anthropometric data of 82 practically healthy men of the same age group.

Statistical processing of body circumference was performed in the licensed package "Statistica 5.5" using non-parametric estimation methods. The reliability of the difference between the values between the independent quantitative values was determined using the U-MannWhitney test.

\section{Results}

Table 1 shows the significant or trends in differences in girth body sizes between healthy and/or psoriatic men of mesomorphic and endo-mesomorphic somatotypes.

\section{Discussion}

Thus, when comparing the girth body sizes in patients with psoriasis Ukrainian men of different somatotypes with healthy men of the corresponding somatotypes, it was found:

- in patients with psoriasis men of mesomorphic 
Table 1. Comparison of body girth between healthy and psoriatic men of mesomorphic and endomosomorphic somatotypes (M $\pm \sigma$ ).

\begin{tabular}{|l|c|c|c|c|c|c|c|c|c|}
\hline \multirow{2}{*}{ Indicators } & \multicolumn{2}{|c|}{ Healthy } & \multirow{2}{*}{$\mathrm{p}$} & \multicolumn{2}{c|}{ Psoriasis $\mathrm{MC}$} & \multirow{2}{*}{$\mathrm{p}$} & \multicolumn{3}{c|}{ Psoriasis SC } \\
& mes. & en-mes. & & mes. & en-mes. & & mes. & en-mes. & $\mathrm{p}$ \\
\hline OBPL1 & $34.97 \pm 2.28$ & $33.35 \pm 1.81$ & $=0.057$ & $36.16 \pm 2.55^{*}$ & $38.63 \pm 1.11 \&$ & $<0.05$ & $36.24 \pm 2.90^{*}$ & $34.72 \pm 4.15$ & $>0.05$ \\
\hline OBPL2 & $31.92 \pm 2.49$ & $30.42 \pm 1.75$ & $=0.095$ & $34.86 \pm 2.43 \#$ & $37.38 \pm 1.60 \&$ & $<0.05$ & $34.94 \pm 2.73 \#$ & $33.83 \pm 3.84$ & $>0.05$ \\
\hline OBPR1 & $28.37 \pm 1.59$ & $27.69 \pm 1.52$ & $>0.05$ & $29.61 \pm 1.78 \&$ & $30.50 \pm 2.31^{*}$ & $>0.05$ & $29.74 \pm 2.16 \#$ & $28.78 \pm 2.25$ & $>0.05$ \\
\hline OBPR2 & $17.96 \pm 1.18$ & $17.31 \pm 1.09$ & $=0.091$ & $18.07 \pm 0.92$ & $19.13 \pm 1.03^{*}$ & $<0.05$ & $18.44 \pm 0.92^{*}$ & $17.56 \pm 1.69$ & $=0.097$ \\
\hline OBK & $21.83 \pm 1.06$ & $21.23 \pm 1.55$ & $>0.05$ & $22.41 \pm 1.19^{*}$ & $23.50 \pm 1.73^{*}$ & $>0.05$ & $22.80 \pm 0.89 \#$ & $22.17 \pm 1.90$ & $>0.05$ \\
\hline OBB & $55.12 \pm 3.85$ & $54.54 \pm 4.03$ & $>0.05$ & $59.82 \pm 4.26 \#$ & $61.88 \pm 6.01^{*}$ & $>0.05$ & $59.60 \pm 5.78 \#$ & $56.50 \pm 6.57$ & $=0.077$ \\
\hline OBBB & $96.67 \pm 5.46$ & $99.15 \pm 3.82$ & $=0.056$ & $103.3 \pm 7.2 \#$ & $109.8 \pm 2.6 \&$ & $<0.05$ & $102.6 \pm 9.1 \#$ & $100.4 \pm 9.1$ & $>0.05$ \\
\hline OBG1 & $37.58 \pm 2.80$ & $37.27 \pm 2.66$ & $>0.05$ & $40.43 \pm 2.18 \#$ & $41.50 \pm 4.36 t$ & $>0.05$ & $40.21 \pm 2.31 \#$ & $37.89 \pm 4.62$ & $=0.076$ \\
\hline OBG2 & $24.04 \pm 1.81$ & $23.85 \pm 2.09$ & $>0.05$ & $25.61 \pm 1.64 \#$ & $25.88 \pm 2.46$ & $>0.05$ & $24.99 \pm 1.61 \&$ & $24.50 \pm 2.09$ & $>0.05$ \\
\hline OBS & $25.06 \pm 1.31$ & $25.46 \pm 1.66$ & $>0.05$ & $25.13 \pm 0.82$ & $26.38 \pm 1.31$ & $=0.056$ & $25.45 \pm 1.08$ & $25.06 \pm 1.69$ & $>0.05$ \\
\hline OBSH & $38.47 \pm 1.71$ & $38.54 \pm 1.57$ & $>0.05$ & $40.13 \pm 2.78 \#$ & $43.00 \pm 1.41 \&$ & $<0.05$ & $41.13 \pm 2.27 \#$ & $40.28 \pm 2.88$ & $>0.05$ \\
\hline OBT & $80.65 \pm 6.11$ & $85.50 \pm 7.15$ & $<0.05$ & $92.93 \pm 15.95 \#$ & $116.1 \pm 12.8 \&$ & $<0.01$ & $98.80 \pm 11.05 \#$ & $97.33 \pm 19.41$ & $>0.05$ \\
\hline OBGK1 & $101.2 \pm 5.2$ & $105.0 \pm 4.3$ & $<0.05$ & $106.6 \pm 8.4 \&$ & $120.9 \pm 7.1 \&$ & $<0.01$ & $108.5 \pm 8.4 \#$ & $109.7 \pm 11.5$ & $>0.05$ \\
\hline OBGK2 & $94.38 \pm 5.21$ & $98.54 \pm 4.18$ & $<0.01$ & $102.5 \pm 8.9 \#$ & $116.5 \pm 6.4 \&$ & $<0.01$ & $104.9 \pm 8.8 \#$ & $105.3 \pm 11.3$ & $>0.05$ \\
\hline OBGK3 & $96.94 \pm 5.60$ & $100.4 \pm 5.2$ & $<0.05$ & $103.9 \pm 8.8 \#$ & $119.0 \pm 6.9 \&$ & $<0.01$ & $106.1 \pm 8.8 \#$ & $107.7 \pm 11.6$ & $>0.05$ \\
\hline
\end{tabular}

Notes: in this and the following table, MC - mild course; SC - severe course; mes. - representtatives of the mesomorphic somatotype; en-mes. - representatives of endo-mesomorphic somatotype; $p$ - the significance of differences in the groups of healthy or sick men between mesomorphs and endo-mesomorphs; *, \&, \# - significant differences (respectively <0.05, <0.01 or <0.001) between the corresponding indicators in the groups of healthy and patients with mild psoriasis mesomorphs or endo-mesomorphs (higher values are noted); *, \&, \# - significant differences (respectively $<0.05,<0.01$ or $<0.001$ ) between the respective indicators in the groups of healthy and psoriasis patients with severe course mesomorphs or endo-mesomorphs (higher values were noted); $t$ - tendency of differences between the corresponding indicators in the groups of healthy and patients with mild psoriasis mesomorphs or endo-mesomorphs (higher indicators are noted); $\mathbf{t}$ - the tendency of differences between the corresponding indicators in groups of healthy and patients with psoriasis of severe course mesomorphs or endo-mesomorphs (higher indicators are noted); significantly $(<0.05)$ higher values of indicators were noted in orange when comparing patients with mild and severe psoriasis according to the corresponding somatotypes (higher indicators were noted); significantly $(<0.01)$ higher values of indicators were noted in red when comparing patients with mild and severe psoriasis according to the corresponding somatotypes (higher indicators were noted); tendencies to higher values of indicators are noted in yellow when comparing between patients with mild and severe psoriasis according to the corresponding somatotypes (higher indicators are noted); OBB - hip girth (cm); OBBB - hips girth (cm); OBG1 - shin girth at the top (cm); OBG2 - shin girth at the bottom (cm); OBGK1 - chest girth on inspiration (cm); OBGK2 - girth of the chest on exhalation (cm); OBGK3 - chest girth at rest (cm); OBK - girth of the hand $(\mathrm{cm})$; OBPL1 - shoulder girth in a tense state $(\mathrm{cm})$; OBPL2 - shoulder girth in the unstressed state (cm); OBPR1 - forearm girth in the upper part (cm); OBPR2 - forearm girth in the lower part (cm); OBS - foot girth (cm); OBSH - neck girth (cm); OBT - waist girth (cm).

somatotype - higher values of shoulder girth in the tense state by $3.3 \%$ and $3.5 \%$ (in the future, respectively, for mild and severe disease), the shoulder in the unstressed state by $8.4 \%$ and $8.6 \%$, forearms in the upper part by $4.2 \%$ and $4.6 \%$, forearms in the lower part by $2.6 \%$ (severe disease only), hands by $2.6 \%$ and $4.3 \%$, thigh by $7.9 \%$ and $7.5 \%$, thighs by $6.4 \%$ and $5.8 \%$, shins at the top by $7.0 \%$ and $6.5 \%$, shins at the bottom by $6.1 \%$ and $3.8 \%$, neck by $4.1 \%$ and $6.5 \%$, waist by $13.2 \%$ and $18.4 \%$, chest on the inhale by $5.1 \%$ and $6.7 \%$, chest on the exhale by $7.9 \%$ and $10.0 \%$, chest at rest by $6.7 \%$ and $8.6 \%$;

- in patients with psoriasis men of endo-mesomorphic somatotype with a mild course of the disease - higher values of the girth of the shoulder in a tense state by $13.7 \%$, shoulder in a relaxed state by $18.6 \%$, forearm in the upper part by $9.2 \%$, forearm in the lower parts by $9.5 \%$, hands by $9.7 \%$, hip by $11.9 \%$, hips by $9.7 \%$, shins in the upper part by
$10.2 \%$, neck by $10.4 \%$, waist by $26.4 \%$, chest on the inhale by $13.2 \%$, chest on the exhale by $15.4 \%$, chest at rest by $15.6 \%$.

It should be noted that in patients with mild and severe psoriasis of men without somatotype, all the girth dimensions (except for the circumference of the foot) are larger than in practically healthy men [17]. When divided into somatotypes, the lack of significant or tendencies of differences in girth body size between healthy and patients with severe psoriasis of endo-mesomorphic somatotype attracts attention.

When comparing the girth body sizes between psoriasis patients Ukrainian men of different somatotypes, it was found:

- in patients with mild psoriasis - greater values in endomesomorphs of the girth of the shoulder in the stressed state by $6.4 \%$, the shoulder in the unstressed state by 
$6.7 \%$, the forearm in the lower part by $5.5 \%$, the thighs by $5.9 \%$, feet by $4.7 \%$, neck by $6.7 \%$, waist by $20.0 \%$, chest on inhalation by $11.8 \%$, chest on exhalation by $12.0 \%$, chest at rest at $12.7 \%$;

- in patients with severe psoriasis - greater values in representatives of the mesomorphic somatotype only the hip circumference by $5.2 \%$ and the shin in the upper part of $5.8 \%$.

It should be noted that only in patients with mild psoriasis in men, differences in body size between representatives of mesomorphic and endo-mesomorphic somatotypes are similar to healthy men, but are more pronounced (see Table 1).

When comparing the girth of the body between patients with psoriasis, Ukrainian men of the corresponding somatotypes found higher values of the girth of the forearm in the upper part by $2.0 \%$, neck by $2.4 \%$, waist by $5.9 \%$, chest by inhalation by $1.8 \%$, chest exhalation by $2.3 \%$ and chest at rest by $2.1 \%$ in mesomorphic somatotypes with more severe disease than mild psoriasis, as well as shin girth in the lower part by $2.4 \%$ in representatives of the mesomorphic somatotype with a mild course of the disease than with a severe course of psoriasis.

It should be noted that when comparing the girth body sizes between patients with psoriasis with mild and severe psoriasis in Ukrainian men without somatotype, only higher values of shin girth in the lower part were found in patients with mild psoriasis [17].

As for the results of foreign studies, their results mostly concern only such indicators as body mass index, waist circumference and body weight as markers of risk of psoriasis. Analysis of the scientometric databases PubMed and Embase revealed that the relative risk of psoriasis with an increase in BMI by 5 units was 1.19 (95\% Cl 1.10-1.28, $\mathrm{I} 2=83 \%, \mathrm{n}=7$ ), and with an increase waist circumference by $10 \mathrm{~cm}$ was 1.37 (95\% Cl 1.23-1.53, I2=0\%, p=0.93, $\mathrm{n}=3$ ) [3].

Dai Y. X. and co-authors [7] conducted long-term followup (from 2001 to 2017) on 60,136 people, of whom 406 developed psoriasis during this time. Statistical analysis of the data collected by the researchers revealed that the risk factor for psoriasis increases with increasing $\mathrm{BMI}$ of the human body (from aHR 1.34 at BMI 25.0-29.9 to 2.70 at $\mathrm{BMI} \geq 30$ ).

33,734 th most common a Norwegian resident took part in a 10-year study to study psoriasis. 369 of the subjects developed psoriasis during the experiment. Compared with obese individuals, obese individuals had an RR of psoriasis of 1.87 (95\% Cl=1.38-2.52) [20].

A similar study, which also conducted long-term followup assessments of anthropometric indicators (from 2009 to 2012), but on a larger scale (estimated data from

\section{References}

[1] Armstrong, A. W., Schupp, C., Wu, J., \& Bebo, B. (2012). Quality of life and work productivity impairment among psoriasis patients: findings from the National Psoriasis Foundation survey data 2003-2011. PloSone, 7(12), e52935. doi: 10.1371/
$22,633,536$ people, of whom 399,461 had newly diagnosed psoriasis) found an increased risk of psoriasis in people with BMI over 30 (HR was 1.118). Similarly, the same risk was found in people with a waist circumference of more than $105 \mathrm{~cm}(\mathrm{HR}, 1.305)$ [10].

Of the 8,016,907 people who underwent three medical examinations from 2010 to $2015,187,128$. persons (ie $2.33 \%$ ) developed psoriasis. As in previous studies, a higher risk of psoriasis was found in people with the highest body weight [13]. All these data are rightly consistent with the results of the previously described work.

Li W. Q. and others [14] have shown that as a result of weight change, the association between IL12B gene polymorphism and the risk of psoriasis may be altered.

Analysis of a sample of 1.5 million people in the United States found that the incidence of psoriasis among normal weight individuals was 11.9 per 10,000 person-years, among overweight people - 11.9, in obese patients - class $1-14.2$, and 17.4 in class 2 and 3 obese patients. The overall incidence of psoriasis was 9.5 per 10,000 personyears [16].

According to the data of the above and other studies, indeed [21, 24], anthropometric indicators can and should be used as markers to identify individuals who have a potentially higher risk of psoriasis than other citizens. Early detection of such individuals would allow the use of lifemodifying factors in order to avoid triggers that cause the development of the disease and increase its severity.

\section{Conclusion}

1. In patients with mild and severe psoriasis Ukrainian men of mesomorphic somatotype, almost all girth dimensions (except for the girth of the foot and forearm in the lower part only in patients with mild psoriasis) are larger than in healthy men; and in patients with psoriasis, Ukrainian men of endo-mesomorphic somatotype larger than in healthy men, almost all the girth dimensions (except for the circumference of the foot and shin in the lower part) only in representatives with mild psoriasis.

2. Men of endo-mesomorphic somatotype with mild psoriasis have higher values of most girth body sizes (except for the forearms in the upper part, hands, thighs and shin) than in representatives of the mesomorphic somatotype; and in patients with severe psoriasis, only the girth of the thigh and shin in men of mesomorphic somatotype is greater than in endo-mesomorphs.

3. In men of mesomorphic somatotype with severe psoriasis, compared with mild psoriasis, higher values of the girth of the forearm in the upper part, neck, waist and all the girth of the chest, as well as lower values of the girth of the shin were found.

\section{journal.pone. 0052935}

[2] Augustin, M., Kruger, K., Radtke, M. A., Schwippl, I., \& Reich, K. (2008). Disease severity, quality of life and health care in plaque-type psoriasis: a multicenter cross-sectional study in 
Germany. Dermatology, 216(4), 366-372. doi: 10.1159/ 000119415

[3] Aune, D., Snekvik, I., Schlesinger, S., Norat, T., Riboli, E., \& Vatten, L. J. (2018). Body mass index, abdominal fatness, weight gain and the risk of psoriasis: a systematic review and dose-response meta-analysis of prospective studies. European journal of epidemiology, 33(12), 1163-1178. doi: 10.1007/s10654-018-0366-z

[4] Bunak, V. V. (1941). Antropometriia [Anthropometry]. M.: Narkompros RSFSR - M.: People's Commissariat of the RSFSR.

[5] Capon, F. (2017). The genetic basis of psoriasis. International journal of molecular sciences, 18(12), 2526. doi: 10.3390/ ijms 18122526

[6] Carter, J. (2003). The Heath-Carter antropometric somatotype. Instruction manual. Department of Exercise and Nutritional Sciences San Diego State University. CA. U.S.A.

[7] Dai, Y. X., Shen, Y. J., Chou, Y. J., Chang, Y. T., Chen, T. J., Li, C. P., \& Wu, C. Y. (2020). Obesity, but not metabolic diseases, is associated with risk of psoriasis: a population-based cohort study in Taiwan. Dermatology, 236(6), 521-528. doi: 10.1159/ 000508303

[8] Enamandram, M., \& Kimball, A. B. (2013). Psoriasis epidemiology: the interplay of genes and the environment. Journal of Investigative Dermatology, 133(2), 287-289. doi: 10.1038/ jid.2012.434

[9] Fredriksson, T., \& Pettersson, U. (1978). Severe psoriasis - oral therapy with a new retinoid. Dermatology, 157(4), 238-244. doi: 10.1159/000250839

[10] Han, J. H., Lee, J. H., Han, K. D., Kim, H. N., Bang, C. H., Park, Y. M., ... \& Kim, T. Y. (2019). Increased risk of psoriasis in subjects with abdominal obesity: A nationwide populationbased study. The Journal of dermatology, 46(8), 695-701. doi: 10.1111/1346-8138.14939

[11] Heller, M. M., Lee, E. S., \& Koo, J. Y. (2011). Stress as an influencing factor in psoriasis. Skin therapy letter, 16(5), 1-4. PMID: 21611682

[12] Hrehorow, E., Salomon, J., Matusiak, U., Reich, A., \& Szepietowski, J. C. (2012). Patients with psoriasis feel stigmatized. Acta dermato-venereologica, 92(1), 67-72.

[13] Kim, M., Han, K. D., \& Lee, J. H. (2020). Bodyweight variability and the risk of psoriasis: a nationwide population-based cohort study. Journal of the European Academy of Dermatology and Venereology, 34(5), 1019-1025. doi: 10.1111/jdv.16099

[14] Li, W. Q., Han, J. L., Zhang, M. F., \& Qureshi, A. A. (2013). Interactions between adiposity and genetic polymorphisms on the risk of psoriasis. British Journal of Dermatology, 168(3),
639-642. doi: 10.1111/bjd.12001

[15] Michalek, I. M., Loring, B., \& John, S. M. (2017). A systematic review of worldwide epidemiology of psoriasis. Journal of the European Academy of Dermatology and Venereology, 31(2), 205-212. doi: 10.1111/jdv.13854

[16] Norden, A., Rekhtman, S., Strunk, A., \& Garg, A. (2021). Risk of psoriasis according to body mass index: a retrospective cohort analysis. Journal of the American Academy of Dermatology. doi: 10.1016/j.jaad.2021.06.012

[17] Obadeh Bassam Abdel-Rahman Al-Qaraleh, Dmytrenko, S. V., Shayuk, A. V., Gunas, I. V., \& Prokopenko S. V. (2020). Peculiarities of girth body sizes in men with mild and severe psoriasis course. Reports of Morphology, 26(4), 62-67. doi: 10.31393/morphology-journal-2020-26(4)-10

[18] Parisi, R., Symmons, D. P., Griffiths, C. E., \& Ashcroft, D. M. (2013). Global epidemiology of psoriasis: a systematic review of incidence and prevalence. Journal of Investigative Dermatology, 133(2), 377-385. doi: 10.1038/jid.2012.339

[19] Rachakonda, T. D., Schupp, C. W., \& Armstrong, A. W. (2014). Psoriasis prevalence among adults in the United States. Journal of the American Academy of Dermatology, 70(3), 512-516. doi: 10.1016/j.jaad.2013.11.013

[20] Snekvik, I., Smith, C. H., Nilsen, T. I., Langan, S. M., Modalsli, E. H., Romundstad, P. R., \& Saunes, M. (2017). Obesity, waist circumference, weight change, and risk of incident psoriasis: prospective data from the HUNT study. Journal of Investigative Dermatology, 137(12), 2484-2490. doi: 10.1016/ j.jid.2017.07.822

[21] Toussirot, E., Aubin, F., \& Dumoulin, G. (2014). Relationships between adipose tissue and psoriasis, with or without arthritis. Frontiers in immunology, 5, 368. doi: 10.3389/ fimmu.2014.00368

[22] Tsai, T. F., Wang, T. S., Hung, S. T., Phiona, I., Tsai, C., Schenkel, B., ... \& Tang, C. H. (2011). Epidemiology and comorbidities of psoriasis patients in a national database in Taiwan. Journal of dermatological science, 63(1), 40-46. doi: 10.1016/ j.jdermsci.2011.03.002

[23] Vanderpuye-Orgle, J., Zhao, Y., Lu, J., Shrestha, A., Sexton, A., Seabury, S., \& Lebwohl, M. (2015). Evaluating the economic burden of psoriasis in the United States. Journal of the American Academy of Dermatology, 72(6), 961-967. doi: 10.1016/j.jaad.2015.02.1099

[24] Zachariae, C., \& Skov, L. (2020). Obesity as a risk factor for psoriasis. Journal of the European Academy of Dermatology and Venereology, 34(5), 915-916. doi: 10.1111/jdv.16434 